\title{
Efficient Iterative Solutions to General Coupled Matrix Equations
}

\author{
Masoud Hajarian \\ Department of Mathematics, Faculty of Mathematical Sciences, Shahid Beheshti University, General Campus, Evin, Tehran 19839, Iran
}

\begin{abstract}
Linear matrix equations are encountered in many systems and control applications. In this paper, we consider the general coupled matrix equations (including the generalized coupled Sylvester matrix equations as a special case) $\sum_{t=1}^{l} E_{s t} Y_{t} F_{s t}=G_{s}, s=$ $1,2, \cdots, l$ over the generalized reflexive matrix group $\left(Y_{1}, Y_{2}, \cdots, Y_{l}\right)$. We derive an efficient gradient-iterative (GI) algorithm for finding the generalized reflexive solution group of the general coupled matrix equations. Convergence analysis indicates that the algorithm always converges to the generalized reflexive solution group for any initial generalized reflexive matrix group $\left(Y_{1}(1), Y_{2}(1), \cdots, Y_{l}(1)\right)$. Finally, numerical results are presented to test and illustrate the performance of the algorithm in terms of convergence, accuracy as well as the efficiency.
\end{abstract}

Keywords: General coupled matrix equations, Lyapunov matrix equation, Sylvester matrix equation, generalized reflexive solution group, iterative algorithm.

\section{Introduction}

The solution for matrix equations plays an important role in matrix computation ${ }^{[1-4]}$. Therefore, various methods for the solution of matrix equations have been investigated ${ }^{[5-10]}$. Many problems in systems and control theory require the solution of generalized Sylvester matrix equation

$$
A X B+C Y D=E .
$$

Wang et al. ${ }^{[11]}$ presented the necessary and sufficient conditions for the existence and an representation of the real and pure imafinary solutions to the consistent quaternion matrix equation (1). Wang ${ }^{[12,13]}$ investigated (1) over an arbitrary division ring and any regular ring with identity. Huang and Zeng ${ }^{[14]}$ considered the matrix equation (1) over a simple Artinian ring. The perturbation analysis of generalized Sylvester eigenspaces of matrix quadruples ${ }^{[15]}$ leads to pairs of generalized Sylvester equations of the form

$$
\begin{aligned}
& A_{1} X-Y B_{1}=C_{1} \\
& A_{2} X-Y B_{2}=C_{2} .
\end{aligned}
$$

Wimmer ${ }^{[16]}$ studied the generalized Sylvester equations (2). Zhou et al. ${ }^{[17]}$ developed a simple and efficient iterative method for solving a class of coupled linear matrix equations which have wide applications in linear system theory. In [18], the coupled discrete-time Markovian jump Lyapunov matrix equations were considered and a simple and effective iterative method to produce numerical solutions to this class of matrix equations was proposed. By adopting the gradient search principle in optimization theory, Zhou et al. ${ }^{[19]}$ provided a general gradient based iterative algorithm to solve the problem of weighted least square solutions for general coupled Sylvester matrix equations. In [20], the closed form solutions to a family of generalized Sylvester matrix equations were given by using the so-called

\footnotetext{
Manuscript received November 6, 2012; revised March 1, 2013
}

Kronecker matrix polynomials. Zhou et al. ${ }^{[21]}$ analyzed the computational complexity of the Smith iteration and its variations for solving Stein matrix equation.

Ding et al. ${ }^{[22]}$ presented a set of gradient-iterative (GI) algorithms of solving the generalized Sylvester matrix equations by using the Jacobi method and hierarchical identification principle ${ }^{[23,24]}$. In [25], a general family of iterative methods was proposed to solve linear equations, which includes the well-known Jacobi and Gauss-Seidel iterations as its special cases. Zhou and Duan ${ }^{[26]}$ presented the general parametric solution to a family of generalized Sylvester matrix equations by using the so-called generalized Sylvester mapping which has some elegant properties. Borno ${ }^{[27]}$ proposed a parallel method for solving the coupled Lyapunov equations. Ding and Chen ${ }^{[28]}$ studied gradient iterative algorithms for solving Sylvester coupled matrix equations and general coupled matrix by using the gradient search principle. In [29], Ding and Chen applied a hierarchical identification principle for solving Sylvester and Lyapunov matrix equations. Recently, Dehghan and Hajarian ${ }^{[30-32]}$ proposed efficient iterative methods based on the conjugate gradient $(\mathrm{CG})$ method for solving several linear matrix equations.

Chen ${ }^{[33]}$ proposed a special class of rectangular matrices of $A$ that has the relation

$$
A=P A Q, A \in \mathbf{R}^{n \times m}
$$

where $P$ and $Q$ are two generalized reflection matrices. The investigation indicated that generalized reflexive matrices arise naturally from problems with reflexive symmetry, which account for a great number of real-world scientific and engineering applications ${ }^{[33]}$. Much of the activity in this field involves exploiting the underlying mathematical or physical problem. These matrices can be defined as follows ${ }^{[33]}$.

Definition 1. Matrix $P \in \mathbf{R}^{n \times n}$ is called a generalized reflection matrix if $P=P^{\mathrm{T}}$ and $P^{2}=I_{n}$. Suppose that $P$ and $Q$ are two generalized reflection matrices of dimensions 
$n$ and $m$, respectively. Matrix $A \in \mathbf{R}^{n \times m}$ is said to be generalized reflexive matrix with respect to matrix pair $(P ; Q)$ if $A=P A Q . \mathbf{R}_{(P ; Q)}^{n \times m}$ denotes the set of order $n \times m$ generalized reflexive with respect to matrix pair $(P ; Q)$. It is obvious that every $n \times m$ matrix is also generalized reflexive with respect to matrix pair $\left(I_{n} ; I_{m}\right)$.

The main concern of this paper is to study the problem of solving the general coupled matrix equations. This problem is considered as follows.

Problem 1. For given matrices $E_{s t} \in \mathbf{R}^{p_{s} \times m_{t}}, F_{s t} \in$ $\mathbf{R}^{n_{t} \times q_{s}}, G_{t} \in \mathbf{R}^{p_{t} \times q_{t}}, P_{t} \in \mathbf{G R}^{m_{t} \times m_{t}}$ and $Q_{t} \in \mathbf{G R}^{n_{t} \times n_{t}}$ $(s, t=1,2, \cdots, l)$, find $\left(Y_{1}, Y_{2}, \cdots, Y_{l}\right)$ with $Y_{t} \in \mathbf{R}_{\left(P_{t} ; Q_{t}\right)}^{m_{t} \times n_{t}}$ $(t=1,2, \cdots, l)$ such that

$$
\sum_{t=1}^{l} E_{s t} Y_{t} F_{s t}=G_{s}, s=1,2, \cdots, l
$$

The paper is organized as follows. In Section 2, we first presented some intermediate results. Then, we propose a GI algorithm for solving Problem 1. We prove that the algorithm consistently converges to the generalized reflexive solution group of Problem 1 for any initial generalized reflexive matrix group. Section 3 illustrates the performance of the algorithm with numerical results. We give some brief conclusions in Section 4 to end this paper.

Notations. $\mathbf{R}^{m \times n}$ : The set of all $m \times n$ real matrices; $\mathbf{G R}^{n \times n}:$ The set of all $n \times n$ generalized reflection matrices; $A^{\mathrm{T}}$ : The transpose of matrix $A$;

$R(A)$ : The column space of matrix $A$;

$\lambda_{\max }(A)$ : The maximum eigenvalue of $A$;

$I_{n}$ : Identity matrix of order $n$;

$A \otimes B:$ Kronecker product of $A$ and $B$;

$\operatorname{tr}(A)$ : The trace of matrix $A$;

$\operatorname{vec}(A): m n \times 1$ vector formed by the vertical concatenation of the respective columns of matrix $A \in \mathbf{R}^{m \times n}$;

$\|A\|=\sqrt{\operatorname{tr}\left(A^{\mathrm{T}} A\right)}$ : Frobenius norm of matrix $A$.

\section{Main results}

In this section, we will derive the solvability conditions for Problem 1. Then, we propose an iterative algorithm for finding the generalized reflexive solution group of Problem 1. In the end, we show that the iterative solution consistently converges to the generalized reflexive solution group of Problem 1 for any initial generalized reflexive matrix group.

It is not difficult to obtain that Problem 1 has a generalized reflexive solution group if and only if the following system of matrix equations is consistent:

$$
\begin{cases}\sum_{t=1}^{l} E_{s t} Y_{t} F_{s t}=G_{s}, & s=1,2, \cdots, l \\ \sum_{t=1}^{l} E_{s t} P_{t} Y_{t} Q_{t} F_{s t}=G_{s}, & s=1,2, \cdots, l .\end{cases}
$$

By considering the above system, the matrix

$$
S=\left(\begin{array}{cccc}
F_{11}^{\mathrm{T}} \otimes E_{11} & F_{12}^{\mathrm{T}} \otimes E_{12} & \cdots & F_{1 l}^{\mathrm{T}} \otimes E_{1 l} \\
F_{11}^{\mathrm{T}} Q_{1} \otimes E_{11} P_{1} & F_{12}^{\mathrm{T}} Q_{2} \otimes E_{12} P_{2} & \cdots & F_{1 l}^{\mathrm{T}} Q_{l} \otimes E_{1 l} P_{l} \\
F_{21}^{\mathrm{T}} \otimes E_{21} & F_{22}^{\mathrm{T}} \otimes E_{22} & \cdots & F_{2 l}^{\mathrm{T}} \otimes E_{2 l} \\
F_{21}^{\mathrm{T}} Q_{1} \otimes E_{21} P_{1} & F_{22}^{\mathrm{T}} Q_{2} \otimes E_{22} P_{2} & \cdots & F_{2 l}^{\mathrm{T}} Q_{l} \otimes E_{2 l} P_{l} \\
\cdots & & \cdots & \cdots \\
F_{l 1}^{\mathrm{T}} \otimes E_{l 1} & F_{l 2}^{\mathrm{T}} \otimes E_{l 2} & \cdots & F_{l l}^{\mathrm{T}} \otimes E_{l l} \\
F_{l 1}^{\mathrm{T}} Q_{1} \otimes E_{l 1} P_{1} & F_{l 2}^{\mathrm{T}} Q_{2} \otimes E_{l 2} P_{2} & \cdots & F_{l l}^{\mathrm{T}} Q_{l} \otimes E_{l l} P_{l}
\end{array}\right),
$$

and the vector

$$
b=\left(\begin{array}{c}
\operatorname{vec}\left(G_{1}\right) \\
\operatorname{vec}\left(G_{1}\right) \\
\operatorname{vec}\left(G_{2}\right) \\
\operatorname{vec}\left(G_{2}\right) \\
\cdots \\
\operatorname{vec}\left(G_{l}\right) \\
\operatorname{vec}\left(G_{l}\right)
\end{array}\right),
$$

we have the following well-known theorem.

Theorem 1. The general coupled matrix equations (3) have a unique generalized reflexive solution group if and only if $\operatorname{rank}(S, b)=\operatorname{rank}(S)$, and $S$ has a full column rank. In this case, the generalized reflexive solution group $\left(Y_{1}^{*}, Y_{2}^{*}, \cdots, Y_{l}^{*}\right)$ of the general coupled matrix equations (3) can be expressed by

$$
\begin{aligned}
& Y_{s}^{*}=\frac{Y_{s}+P_{s} Y_{s} Q_{s}}{2}, \quad s=1,2, \cdots, l \\
& \left(\begin{array}{c}
\operatorname{vec}\left(Y_{1}\right) \\
\operatorname{vec}\left(Y_{2}\right) \\
\cdots \\
\operatorname{vec}\left(Y_{l}\right)
\end{array}\right)=\left(S^{\mathrm{T}} S\right)^{-1} S^{\mathrm{T}} b .
\end{aligned}
$$

And the homogenous general coupled matrix equations

$$
\sum_{t=1}^{l} E_{s t} Y_{t} F_{s t}=0, \quad s=1,2, \cdots, l
$$

have a unique generalized reflexive solution group $\left(Y_{1}, Y_{2}, \cdots, Y_{l}\right)=0$.

In the expressed solution (6), the dimension of coefficient matrix $S$ has increased geometrically, and a matrix inversion is required which may turn out to be numerically expensive. To overcome these complications and drawbacks, we extend the idea of the Jacobi and the Gauss-Seidel iterations for solving Problem 1.

Consider a linear algebraic system of equations

$$
A x=b
$$

where $A \in \mathbf{R}^{n \times n}$ is the coefficient matrix, $b$ and $x$ are the known right hand side and the solution to be sought. Also, suppose

$$
A=D-E-F
$$


in which $D$ is the diagonal of $A,-E$ is its strict lower part, and $-F$ is its strict upper part. It is always assumed that the diagonal entries of $A$ are all nonzero. To solve the linear system (8), the Jacobi and the Gauss-Seidel iterations are both of the form

$$
M x(k+1)=N x(k)+b=(M-A) x(k)+b
$$

in which

$$
A=M-N
$$

is a splitting of $A$, with $M=D$ for Jacobi, $M=D-E$ for forward Gauss-Seidel, and $M=D-F$ for backward Gauss-Seidel. Now, by extending the Jacobi and the GaussSeidel iterations and applying the hierarchical identification principle $^{[22,28]}$, we propose an algorithm to solve the general coupled matrix equations (3) over the generalized reflexive matrix group $\left(Y_{1}, Y_{2}, \cdots, Y_{l}\right)$.

Algorithm 1. GI algorithm

Step 1. Choose an initial generalized reflexive matrix group $\left(Y_{1}(1), Y_{2}(1), \cdots, Y_{l}(1)\right)$ with $Y_{t}(1) \in \mathbf{R}_{\left(P_{t} ; Q_{t}\right)}^{m_{t} \times n_{t}}(t=$ $1,2, \cdots, l)$ and a parameter $\delta>0$.

Step 2. Compute

$$
R_{s}(1)=G_{s}-\sum_{t=1}^{l} E_{s t} Y_{t}(1) F_{s t}, \quad s=1,2, \cdots, l .
$$

Step 3. For $k=1,2, \cdots$, compute

$$
\begin{aligned}
& Y_{t}(k+1)=Y_{t}(k)+ \\
& \frac{\delta}{2}\left[\sum_{s=1}^{l}\left(E_{s t}^{\mathrm{T}} R_{s}(k) F_{s t}^{\mathrm{T}}+P_{t} E_{s t}^{\mathrm{T}} R_{s}(k) F_{s t}^{\mathrm{T}} Q_{t}\right)\right],
\end{aligned}
$$

$t=1,2, \cdots, l$

and

$$
R_{s}(k+1)=G_{s}-\sum_{t=1}^{l} E_{s t} Y_{t}(k+1) F_{s t}, \quad s=1,2, \cdots, l .
$$

Remark 1. From Algorithm 1, we can easily see that $Y_{t}(k) \in \mathbf{R}_{\left(P_{t} ; Q_{t}\right)}^{m_{t} \times n_{t}}$ for $t=1,2, \cdots, l$ and $k=1,2, \cdots$.

In Theorem 2, we will establish the convergence theory of Algorithm 1.

Theorem 2. Suppose that the general coupled matrix equations (3) have a unique generalized reflexive solution group $\left(Y_{1}^{*}, Y_{2}^{*}, \cdots, Y_{l}^{*}\right)$. If parameter $\delta$ satisfies the inequality

$$
0<\delta<\frac{2}{\sum_{s=1}^{l} \sum_{t=1}^{l}\left\|E_{s t} F_{s t}\right\|^{2}}
$$

then for any initial generalized reflexive matrix group $\left(Y_{1}(1), Y_{2}(1), \cdots, Y_{l}(1)\right)$ with $Y_{t}(1) \quad \in$ $\mathbf{R}_{\left(P_{t} ; Q_{t}\right)}^{m_{t} \times n_{t}} \quad(t=1,2, \cdots, l)$, the iterative solution $\left(Y_{1}(k), Y_{2}(k), \cdots, Y_{l}(k)\right)$ generated by Algorithm 1 converges to the $\left(Y_{1}^{*}, Y_{2}^{*}, \cdots, Y_{l}^{*}\right)$, i.e.,

$$
\lim _{k \rightarrow \infty} Y_{t}(k)=Y_{t}^{*}, \quad t=1,2, \cdots, l .
$$

Proof. Assume that

$$
\tilde{Y}_{t}(k)=Y_{t}(k)-Y_{t}^{*}, \quad t=1,2, \cdots, l
$$

denote the error matrices in the $k$-th iteration of Algorithm 1 , which produces the sequences $\left\{Y_{t}(k)\right\}$ for $t=1,2, \cdots, l$ and $k=1,2, \cdots$. It is not difficult to see that $\widetilde{Y}_{t}(k) \in$ $\mathbf{R}_{\left(P_{t} ; Q_{t}\right)}^{m_{t} \times n_{t}}$ for $t=1,2, \cdots, l$. Also, we can write

$$
R_{t}(k)=-\sum_{j=1}^{l} E_{t j} \tilde{Y}_{j}(k) F_{t j}
$$

and

$$
\begin{gathered}
\tilde{Y}_{t}(k+1)=\tilde{Y}_{t}(k)-\frac{\delta}{2}\left[\sum_{s=1}^{l} E_{s t}^{\mathrm{T}}\left(\sum_{j=1}^{l} E_{s j} \tilde{Y}_{j}(k) F_{s j}\right) F_{s t}^{\mathrm{T}}+\right. \\
\left.\sum_{s=1}^{l} P_{t} E_{s t}^{\mathrm{T}}\left(\sum_{j=1}^{l} E_{s j} \tilde{Y}_{j}(k) F_{s j}\right) F_{s t}^{\mathrm{T}} Q_{t}\right]
\end{gathered}
$$

for $t=1,2, \cdots, l$. It is well known that $\|P A Q\|=\|A\|$ for any appropriately dimensioned generalized reflection matrices $P$ and $Q$. Accordingly, we have

$$
\begin{aligned}
& \left\|\widetilde{Y}_{t}(k+1)\right\|^{2}=\operatorname{tr}\left(\tilde{Y}_{t}(k+1)^{\mathrm{T}} \widetilde{Y}_{t}(k+1)\right)= \\
& \left\|\widetilde{Y}_{t}(k)\right\|^{2}-\delta \operatorname{tr}\left(\tilde { Y } _ { t } ( k ) ^ { \mathrm { T } } \left[\sum_{s=1}^{l} E_{s t}^{\mathrm{T}}\left(\sum_{j=1}^{l} E_{s j} \tilde{Y}_{j}(k) F_{s j}\right) F_{s t}^{\mathrm{T}}+\right.\right. \\
& \left.\left.\sum_{s=1}^{l} P_{t} E_{s t}^{\mathrm{T}}\left(\sum_{j=1}^{l} E_{s j} \tilde{Y}_{j}(k) F_{s j}\right) F_{s t}^{\mathrm{T}} Q_{t}\right]\right)+ \\
& \frac{\delta^{2}}{4} \| \sum_{s=1}^{l} E_{s t}^{\mathrm{T}}\left(\sum_{j=1}^{l} E_{s j} \tilde{Y}_{j}(k) F_{s j}\right) F_{s t}^{\mathrm{T}}+ \\
& \sum_{s=1}^{l} P_{t} E_{s t}^{\mathrm{T}}\left(\sum_{j=1}^{l} E_{s j} \tilde{Y}_{j}(k) F_{s j}\right) F_{s t}^{\mathrm{T}} Q_{t} \|^{2} \leqslant \\
& \left\|\tilde{Y}_{t}(k)\right\|^{2}-\delta \operatorname{tr}\left(\sum_{s=1}^{l} E_{s t} \tilde{Y}_{t}(k) F_{s t}\left(\sum_{j=1}^{l} E_{s j} \tilde{Y}_{j}(k) F_{s j}\right)^{\mathrm{T}}+\right. \\
& \left.\sum_{s=1}^{l} E_{s t} P_{t} \tilde{Y}_{t}(k) Q_{t} F_{s t}\left(\sum_{j=1}^{l} E_{s j} \tilde{Y}_{j}(k) F_{s j}\right)^{\mathrm{T}}\right)+ \\
& \frac{\delta^{2}}{2}\left[\left\|\sum_{s=1}^{l} E_{s t}^{\mathrm{T}}\left(\sum_{j=1}^{l} E_{s j} \tilde{Y}_{j}(k) F_{s j}\right) F_{s t}^{\mathrm{T}}\right\|^{2}+\right. \\
& \left.\left\|\sum_{s=1}^{l} P_{t} E_{s t}^{\mathrm{T}}\left(\sum_{j=1}^{l} E_{s j} \tilde{Y}_{j}(k) F_{s j}\right) F_{s t}^{\mathrm{T}} Q_{t}\right\|^{2}\right] \leqslant \\
& \left\|\tilde{Y}_{t}(k)\right\|^{2}-2 \delta \operatorname{tr}\left(\sum_{s=1}^{l} E_{s t} \tilde{Y}_{t}(k) F_{s t}\left(\sum_{j=1}^{l} E_{s j} \tilde{Y}_{j}(k) F_{s j}\right)^{\mathrm{T}}\right)+ \\
& \delta^{2}\left(\sum_{s=1}^{l}\left\|E_{s t} F_{s t}\right\|^{2}\right) \sum_{s=1}^{l}\left\|\sum_{j=1}^{l} E_{s j} \tilde{Y}_{j}(k) F_{s j}\right\|^{2}
\end{aligned}
$$

We define the nonnegative definite function $Z(k)$ as

$$
Z(k+1)=\sum_{t=1}^{l}\left\|\widetilde{Y}_{t}(k+1)\right\|^{2}
$$


By applying (17) and (18), we obtain

$$
\begin{aligned}
& Z(k+1)=\sum_{t=1}^{l}\left\|\widetilde{Y}_{t}(k+1)\right\|^{2} \leqslant \sum_{t=1}^{l}\left\|\widetilde{Y}_{t}(k)\right\|^{2}- \\
& 2 \delta \operatorname{tr}\left(\sum_{t=1}^{l} \sum_{s=1}^{l} E_{s t} \widetilde{Y}_{t}(k) F_{s t}\left(\sum_{j=1}^{l} E_{s j} \widetilde{Y}_{j}(k) F_{s j}\right)^{\mathrm{T}}\right)+ \\
& \delta^{2}\left(\sum_{s=1}^{l}\left\|E_{s t} F_{s t}\right\|^{2}\right) \sum_{s=1}^{l}\left\|\sum_{j=1}^{l} E_{s j} \tilde{Y}_{j}(k) F_{s j}\right\|^{2}= \\
& Z(k)-2 \delta \sum_{s=1}^{l}\left\|\sum_{j=1}^{l} E_{s j} \tilde{Y}_{j}(k) F_{s j}\right\|^{2}+ \\
& \delta^{2}\left(\sum_{s=1}^{l}\left\|E_{s t} F_{s t}\right\|^{2}\right) \sum_{s=1}^{l}\left\|\sum_{j=1}^{l} E_{s j} \tilde{Y}_{j}(k) F_{s j}\right\|^{2} \leqslant \\
& Z(0)-\delta\left[2-\delta \sum_{t=1}^{l} \sum_{s=1}^{l}\left\|E_{s t} F_{s t}\right\|^{2}\right] \times \\
& \sum_{m=1}^{\infty} \sum_{s=1}^{l}\left\|\sum_{j=1}^{l} E_{s j} \tilde{Y}_{j}(m) F_{s j}\right\|^{2} .
\end{aligned}
$$

If the convergence factor $\delta$ is chosen to satisfy

$$
0<\delta<\frac{2}{\sum_{s=1}^{l} \sum_{t=1}^{l}\left\|E_{s t} F_{s t}\right\|^{2}}
$$

we get

$$
\sum_{m=1}^{\infty} \sum_{s=1}^{l}\left\|\sum_{j=1}^{l} E_{s j} \tilde{Y}_{j}(m) F_{s j}\right\|^{2}<\infty .
$$

It follows from the necessary condition of the series convergence that

$$
\lim _{m \rightarrow \infty} \sum_{s=1}^{l}\left\|\sum_{j=1}^{l} E_{s j} \widetilde{Y}_{j}(m) F_{s j}\right\|^{2}=0 .
$$

Therefore, we can write

$$
\lim _{m \rightarrow \infty} \sum_{j=1}^{l} E_{s j} \tilde{Y}_{j}(m) F_{s j}=0, \quad s=1,2, \cdots, l .
$$

Now, according to Theorem 1, we have

$$
\lim _{m \rightarrow \infty} \tilde{Y}_{j}(m)=0, \quad j=1,2, \cdots, l .
$$

Remark 2. The convergence factor $\delta$ may also be taken as

$$
0<\delta<\frac{2}{\sum_{s=1}^{l} \sum_{t=1}^{l} \lambda_{\max }\left(E_{s t} E_{s t}^{\mathrm{T}}\right) \lambda_{\max }\left(F_{s t} F_{s t}^{\mathrm{T}}\right)} .
$$

The approach in [17] can be used to determine the optimal step size $\delta$ such that the convergence rate is maximized.

In the next section, an example is given to illustrate the convergence properties of Algorithm 1.

\section{$3 \quad$ Numerical results}

In this section, we will present a numerical example to illustrate our results. All the tests are performed using Matlab 7.14.

Example 1. Consider the generalized coupled Sylvester matrix equations

$$
\left\{\begin{array}{l}
A_{1} X B_{1}+C_{1} Y D_{1}=E_{1} \\
A_{2} X B_{2}+C_{2} Y D_{2}=E_{2}
\end{array}\right.
$$

with the parameters

$$
\begin{aligned}
& A_{1}=\left(\begin{array}{llll}
9.8856 & 0.8913 & 0.8214 & 0.9218 \\
0.2311 & 9.1150 & 0.4447 & 0.7382 \\
0.6068 & 0.4565 & 8.8142 & 0.1763 \\
0.4860 & 0.0185 & 0.7919 & 9.1525
\end{array}\right) \\
& A_{2}=\left(\begin{array}{cccc}
-2.6951 & 0.3200 & 0 & 0 \\
0.4983 & -2.6150 & 0.2679 & 0 \\
0.2140 & 0.7266 & -2.5729 & 0.8392 \\
0.6435 & 0.4120 & 0.9334 & -2.9836
\end{array}\right) \\
& B_{1}=\left(\begin{array}{cccc}
8.7497 & 0.8462 & 0 & 0 \\
0.9318 & 9.0668 & 0.0196 & 0 \\
0.4660 & 0.2026 & 9.5349 & 0.7095 \\
0.4186 & 0.6721 & 0.3795 & 9.0738
\end{array}\right) \\
& B_{2}=\left(\begin{array}{cccc}
-7.0907 & 0.5869 & 0.7176 & 0.4418 \\
0.0158 & -7.3932 & 0.6927 & 0.3533 \\
0.0164 & 0.3676 & -7.7815 & 0.1536 \\
0.1901 & 0.6315 & 0.4544 & -6.5637
\end{array}\right) \\
& C_{1}=\left(\begin{array}{cccc}
8.6128 & 0.3412 & 0 & 0 \\
0.6602 & 8.5138 & 0.5681 & 0 \\
0.3420 & 0.7271 & 7.5069 & 0.6946 \\
0.2897 & 0.3093 & 0.7027 & 8.2828
\end{array}\right) \\
& C_{2}=\left(\begin{array}{cccc}
-9.4088 & 0.8439 & 0.4398 & 0.3932 \\
0.0784 & -9.6990 & 0.3400 & 0.5915 \\
0.6408 & 0.1708 & -9.5562 & 0.1197 \\
0.1909 & 0.9943 & 0.3651 & -9.8522
\end{array}\right) \\
& D_{1}=\left(\begin{array}{cccc}
2.7833 & 0.5828 & 0.4329 & 0.5298 \\
0 & 2.0592 & 0.2259 & 0.6405 \\
0 & 0 & 2.8744 & 0.2091 \\
0 & 0 & 0 & 2.7889
\end{array}\right) \\
& D_{2}=\left(\begin{array}{llll}
9.0323 & 0.1556 & 0.4902 & 0.4507 \\
0.6873 & 8.7438 & 0.8159 & 0.4122 \\
0.3461 & 0.4225 & 8.8367 & 0.9016 \\
0.1660 & 0.8560 & 0.4574 & 8.9255
\end{array}\right) \\
& E_{1}=10^{3} \times\left(\begin{array}{cccc}
1.6267 & 0.3016 & 0.2796 & 0.1611 \\
0.2055 & 1.4373 & 0.0720 & 0.2197 \\
0.2847 & 0.1232 & 1.3815 & 0.1254 \\
0.1666 & 0.1407 & 0.1946 & 1.3950
\end{array}\right) \\
& E_{2}=\left(\begin{array}{cccc}
833.7377 & -106.5381 & 1.0169 & -26.9922 \\
-42.0523 & 802.0204 & -46.0233 & 1.7532 \\
-57.0362 & -91.6367 & 797.5347 & -75.3762 \\
-116.0316 & -141.2919 & -146.7395 & 883.0653
\end{array}\right)
\end{aligned}
$$

The generalized coupled Sylvester matrix equations are 
consistent over the generalized reflexive matrices, and have the generalized reflexive matrix pair $\left(X^{*}, Y^{*}\right)$ as

$$
X^{*}=\left(\begin{array}{cccc}
19.7712 & 0 & 1.6428 & 0 \\
0 & 18.2299 & 0 & 1.4764 \\
1.2137 & 0 & 17.6283 & 0 \\
0 & 0.0370 & 0 & 18.3050
\end{array}\right) \in \mathbf{R}_{(P ; Q)}^{4 \times 4}
$$

and

$Y^{*}=\left(\begin{array}{cccc}-5.3902 & 0 & 0 & 0 \\ 0 & -5.2301 & 0 & 0 \\ 0.4279 & 0 & -5.1459 & 0 \\ 0 & 0.8239 & 0 & -5.9672\end{array}\right) \in \mathbf{R}_{(P ; Q)}^{4 \times 4}$

with

$$
P=Q=\left(\begin{array}{cccc}
-1 & 0 & 0 & 0 \\
0 & 1 & 0 & 0 \\
0 & 0 & -1 & 0 \\
0 & 0 & 0 & 1
\end{array}\right) \in \mathbf{G R}^{4 \times 4}
$$

For this example, we apply Algorithm 1 to compute $X(k)$ and $Y(k)$ with the initial matrices $X(1)=Y(1)=0$. In Fig. 1, we give the obtained results with several values of $\delta$, where

$$
\begin{aligned}
r(k)= & \log _{10}\left[\left\|E_{1}-A_{1} X B_{1}-C_{1} Y D_{1}\right\|^{2}+\right. \\
& \left.\left\|E_{2}-A_{2} X B_{2}-C_{2} Y D_{2}\right\|^{2}\right]^{\frac{1}{2}}
\end{aligned}
$$

and

$$
\delta(k)=\log _{10} \frac{\left\|(X(k), Y(k))-\left(X^{*}, Y^{*}\right)\right\|}{\left\|\left(X^{*}, Y^{*}\right)\right\|} .
$$

It can be observed from Fig. 1 that Algorithm 1 is effective. The effect of changing the convergence factor $\delta$ is illustrated in Fig. 1.

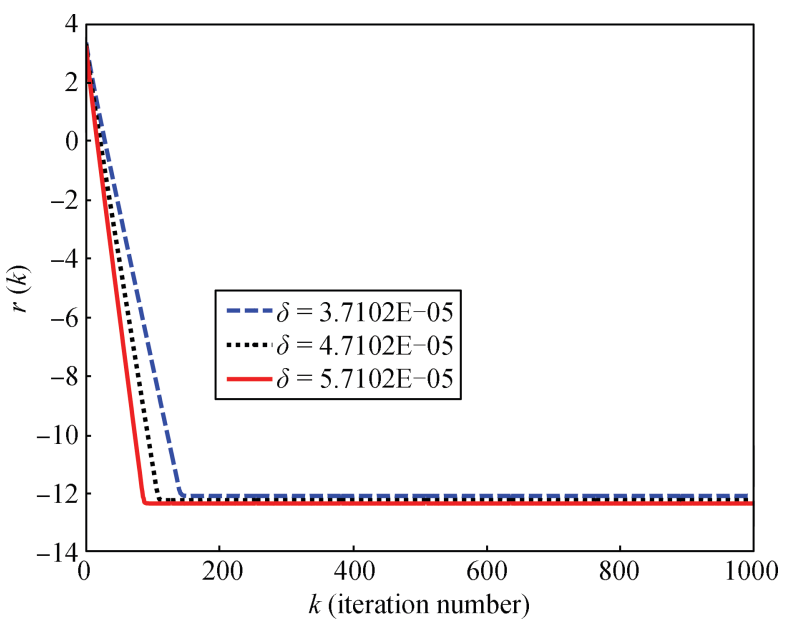

(a)



(b)

Fig. 1 The results obtained for Example 1

\section{Conclusions}

This paper proposes an efficient iterative algorithm for solving the general coupled matrix equations over the generalized reflexive matrices, which have important applications in control theory. Convergence of the iterative algorithm is proved. The results from the numerical example describe the performance of Algorithm 1. We also observe that the accuracy achieved is acceptable.

\section{Acknowledgments}

The author would like to express his heartfelt thanks to the editors and anonymous referees for their useful comments and constructive suggestions.

\section{References}

[1] B. Zhou, G. R. Duan. An explicit solution to the matrix equation $A X-X F=B Y$. Linear Algebra and Its Applications, vol. 402, pp. 345-366, 2005.

[2] B. Zhou, G. R. Duan. A new solution to the generalized Sylvester matrix equation $A V-E V F=B W$. Systems \& Control Letters, vol. 55, no. 3, pp. 193-198, 2006.

[3] B. Zhou, Z. B. Yan. Solutions to right coprime factorizations and generalized Sylvester matrix equations. Transactions of the Institute of Measurement and Control, vol. 30, no. 5, pp. 397-426, 2008.

[4] B. Zhou, G. R. Duan. Closed-form solutions to the matrix equation $A X-E X F=B Y$ with $F$ in companion form. International Journal of Automation and Computing, vol. 6 , no. 2, pp. 204-209, 2009.

[5] M. Dehghan, M. Hajarian. The reflexive and anti-reflexive solutions of a linear matrix equation and systems of matrix equations. Rocky Mountain Journal of Mathematics, vol. 40, no. 3, pp. 825-848, 2010.

[6] M. Dehghan, M. Hajarian. The generalised Sylvester matrix equations over the generalised bisymmetric and skewsymmetric matrices. International Journal of Systems Science, vol. 43, no. 8, pp. 1580-1590, 2012.

[7] M. Dehghan, M. Hajarian. SSHI methods for solving general linear matrix equations. Engineering Computations, vol. 28 , no. 8 , pp. 1028-1043, 2011. 
[8] M. A. Ramadan, E. A. El-Sayed. On the matrix equation $X H=H X$ and the associated controllability problem. Applied Mathematics and Computation, vol.186, no.1, pp. 844-859, 2007.

[9] F. X. Zhang, Y. Li, W. B. Guo, J. L. Zhao. Least squares solutions with special structure to the linear matrix equation $A X B=C$. Applied Mathematics and Computation, vol. 217, no. 24, pp. 10049-10057, 2011.

[10] J. K. Baksalary, P. Kala. The matrix equation AXB + CYD = E. Linear Algebra and Its Applicationa, vol. 30, pp. 141$147,1980$.

[11] Q. W. Wang, H. S. Zhang, S. W. Yu. On solutions to the quaternion matrix equation $\mathrm{AXB}+\mathrm{CYD}=\mathrm{E}$. Electronic Journal of Linear Algebra, vol. 17, pp. 343-358, 2008.

[12] Q. W. Wang. A system of matrix equations and a linear matrix equation over arbitrary regular rings with identity. Linear Algebra and Its Applications, vol. 384, pp. 43-54, 2004.

[13] Q. W. Wang. A system of four matrix equations over von Neumann regular rings and its applications. Acta Mathematica Sinica, vol. 21, no. 2, pp. 323-334, 2005.

[14] L. P. Huang, Q. G. Zeng. The solvability of matrix equation $A X B+C Y D=E$ over a simple Artinian ring. Linear and Multilinear Algebra, vol. 38, no. 3, pp. 225-232, 1995.

[15] J. W. Demmel, B. Kaagstrom. Computing stable eigendecompositions of matrix pencils. Linear Algebra and Its Application, vol. 88-89, pp. 139-186, 1987.

[16] H. K. Wimmer. Consistency of a pair of generalized Sylvester equations. IEEE Transactions on Automatic Control, vol. 39, no. 5, pp. 1014-1016, 1994.

[17] B. Zhou, G. R. Duan, Z. Y. Li. Gradient based iterative algorithm for solving coupled matrix equations. Systems \& Control Letters, vol. 58, no. 5, pp. 327-333, 2009.

[18] B. Zhou, J. Lam, G. R. Duan. Convergence of gradientbased iterative solution of coupled Markovian jump Lyapunov equations. Computers and Mathematics with Applications, vol. 56, no. 12, pp. 3070-3078, 2008.

[19] B. Zhou, Z. Y. Li, G. R. Duan, Y. Wang. Weighted least squares solutions to general coupled Sylvester matrix equations. Journal of Computational and Applied Mathematics, vol. 224, no. 2, pp. 759-776, 2009.

[20] B. Zhou, Z. Y. Li, G. R. Duan, Y. Wang. Solutions to a family of matrix equations by using the Kronecker matrix polynomials. Applied Mathematics and Computation, vol. 212, no. 2, pp. 327-336, 2009.

[21] B. Zhou, J. Lam, G. R. Duan. On Smith-type iterative algorithms for the Stein matrix equation. Applied Mathematics Letters, vol. 22, no. 7, pp. 1038-1044, 2009.

[22] F. Ding, P. X. Liu, J. Ding. Iterative solutions of the generalized Sylvester matrix equations by using the hierarchical identification principle. Applied Mathematics and Computation, vol. 197, no. 1, pp. 41-50, 2008.
[23] F. Ding, T. W. Chen. Hierarchical gradient-based identification of multivariable discrete-time systems. Automatica, vol. 41, no. 2, pp. 315-325, 2005.

[24] F. Ding, T. W. Chen. Hierarchical least squares identification methods for multivariable systems. IEEE Transactions on Automatic Control, vol. 50, no. 3, pp. 397-402, 2005.

[25] F. Ding, T. W. Chen. Iterative least squares solutions of coupled Sylvester matrix equations. Systems \& Control Letters, vol. 54, no. 2, pp. 95-107, 2005.

[26] B. Zhou, G. R. Duan. On the generalized Sylvester mapping and matrix equations. Systems \& Control Letters, vol. 57, no. 3, pp. 200-208, 2008.

[27] I. Borno. Parallel computation of the solutions of coupled algebraic Lyapunov equations. Automatica, vol. 31, no. 9, pp. 1345-1347, 1995.

[28] F. Ding, T. W. Chen. On iterative solutions of general coupled matrix equations. SIAM Journal on Control and Optimization, vol. 44, no. 6, pp. 2269-2284, 2006.

[29] F. Ding, T. W. Chen. Gradient based iterative algorithms for solving a class of matrix equations. IEEE Transactions on Automatic Control, vol. 50, no. 8, pp. 1216-1221, 2005.

[30] M. Dehghan, M. Hajarian. On the generalized bisymmetric and skew-symmetric solutions of the system of generalized Sylvester matrix equations. Linear and Multilinear Algebra, vol. 59, no. 11, pp. 1281-1309, 2011.

[31] M. Dehghan, M. Hajarian. Efficient iterative method for solving the second-order Sylvester matrix equation $E V F^{2}-$ $A V F-C V=B W$. IET Control Theory and Applications, vol. 3, no. 10, pp. 1401-1408, 2009.

[32] M. Dehghan, M. Hajarian. The general coupled matrix equations over generalized bisymmetric matrices. Linear Algebra and Its Applications, vol. 432, no. 6, pp. 1531-1552, 2010

[33] H. C. Chen. Generalized reflexive matrices: special properties and applications. SIAM Journal on Matrix Analysis and Applications, vol. 19, no. 1, pp. 140-153, 1998.

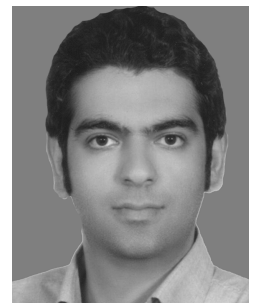

Masoud Hajarian graduated from Department of Mathematics, Shahid Chamran University of Ahvaz, Iran in 2006. He received his $\mathrm{M}$. Sc. and $\mathrm{Ph}$. D. degrees in numerical linear algebra from Amirkabir University of Technology, Iran in 2008 and 2010 , respectively. He is currently working as an assistant professor with the Department of Mathematics, Faculty of Mathematical Sciences, Shahid Beheshti University, General Campus, Iran.

His research interests include numerical linear algebra, matrix theory, numerical analysis and operational research.

E-mail: m_hajarian@sbu.ac.ir 